\title{
On decay rates of the solutions of parabolic Cauchy problems
}

\section{Bonet, José}

2021-06

Bonet, J , Lusky, W \& Taskinen , J 2021, ' On decay rates of the solutions of parabolic

Cauchy problems ' , Proceedings of the Royal Society of Edinburgh. Section A, Mathematics , vol. 151 , no. 3 , 0308210520000487 , pp. 1021-1039 . https://doi.org/10.1017/prm.2020.48

http://hdl.handle.net/10138/331482

https://doi.org/10.1017/prm.2020.48

cc_by_nc_nd

acceptedVersion

Downloaded from Helda, University of Helsinki institutional repository.

This is an electronic reprint of the original article.

This reprint may differ from the original in pagination and typographic detail.

Please cite the original version. 


\title{
ON DECAY RATES OF THE SOLUTIONS OF PARABOLIC CAUCHY PROBLEMS
}

\author{
JOSÉ BONET, WOLFGANG LUSKY, AND JARI TASKINEN
}

\begin{abstract}
We consider the Cauchy problem for a general class of parabolic partial differential equations in the Euclidean space $\mathbb{R}^{N}$. We show that given a weighted $L^{p}$-space $L_{w}^{p}\left(\mathbb{R}^{N}\right)$ with $1 \leq p<\infty$ and a fast growing weight $w$, there is a Schauder basis $\left(e_{n}\right)_{n=1}^{\infty}$ in $L_{w}^{p}\left(\mathbb{R}^{N}\right)$ with the following property: given an arbitrary positive integer $m$ there exists $n_{m}>0$ such that, if the initial data $f$ belongs to the closed linear span of $e_{n}$ with $n \geq n_{m}$, then the decay rate of the solution of the problem is at least $t^{-m}$ for large times $t$.

The result generalizes the recent study of the authors concerning the classical linear heat equation. We present variants of the result having different methods of proofs and also consider finite polynomial decay rates instead of unlimited $m$.
\end{abstract}

\section{INTRODUCTION AND PRELIMINARIES.}

Given an integrable function $f \in L^{1}\left(\mathbb{R}^{N}\right)$ in the Euclidean space $\mathbb{R}^{N}, N \in \mathbb{N}=$ $\{1,2, \ldots\}$, we study the following parabolic Cauchy problem for an unknown function $u$ on $\mathbb{R}^{N} \times[0, \infty) \ni(x, t)$,

$$
\begin{aligned}
\partial_{t} u(x, t) & =A u(x, t) \text { for } x \in \mathbb{R}^{N}, t>0 \\
u(x, 0) & =f(x) \text { for } x \in \mathbb{R}^{N},
\end{aligned}
$$

where $-A$ is a strongly elliptic partial differential operator of $n$th order with even $n \in \mathbb{N}$. More precisely, we assume

$$
-A g(x)=\sum_{|\alpha| \leq n} a_{\alpha}(x) D^{\alpha} g(x),
$$

where the coefficients $a_{\alpha}$ belong to $L^{\infty}\left(\mathbb{R}^{N}\right)$, and the ellipticity condition means that for some constant $c>0$

$$
(-1)^{n / 2} \operatorname{Re} \sum_{|\alpha|=n} a_{\alpha}(x) \xi^{\alpha} \geq c|\xi|^{n} \forall x, \xi \in \mathbb{R}^{N} .
$$

(For elliptic partial differential operators, see [2], Sect. 6.1, [4], Ch. I,V, [6], [7], Ch. 7, or many others; for the notation, see below). In addition, we make the assumptions that $A$ is a generator of a $C_{0}$-semigroup $e^{A t}$, see [7], with an integral kernel $K$ : $\mathbb{R}^{N} \times \mathbb{R}^{N} \times[0, \infty) \rightarrow \mathbb{C}$

$$
e^{A t} f(x)=\int_{\mathbb{R}^{N}} K(x, y, t) f(y) d y, \quad x \in \mathbb{R}^{N},
$$

1991 Mathematics Subject Classification. 35K05, 35B40,46B15,46N20.

Key words and phrases. Parabolic PDE; Cauchy problem; Banach space; Schauder basis; decay rate.

Acknowledgement: The research of Bonet was partially supported by the projects MTM201676647-P and GV Prometeo/2017/102 (Spain). The research of Taskinen was partially supported by a research grant from the Faculty of Science of the University of Helsinki. 
and that the problem (1.1)-(1.2) has a unique classical solution which coincides with (1.5). We assume that the kernel $K$ is a Lebesgue measurable function such that the integral (1.5) converges for all $f \in L^{1}\left(\mathbb{R}^{N}\right)$ and almost all $x, t$. All of our further, crucial assumptions will be imposed in the sequel directly to the kernel $K$. In fact, the proofs of our main results will not even use the assumption that $A$ is a partial differential operator, although our main examples will be such operators.

In the paper [1] we showed that given a rapidly increasing weight $w: \mathbb{R} \rightarrow(0, \infty)$ satisfying some technical assumptions, one can find a Schauder basis $\left(e_{n}\right)_{n=1}^{\infty}$ of the Banach-space $L_{w}^{p}\left(\mathbb{R}^{N}\right), 1 \leq p<\infty$, with the following property: given any $m \in \mathbb{N}$, the solution of the heat equation, i.e. the problem (1.1)-(1.2) for the Laplacian $A=\Delta$, satisfies the decay estimate

$$
\left\|e^{t \Delta} f\right\|_{\infty} \leq \frac{C}{(1+t)^{m}}, \quad t>0
$$

for all initial data $f$ in the closed linear span of the basis elements $e_{n}$ with $n \geq n_{m}$ for some $n_{m} \in \mathbb{N}$. In other words, the subspace of initial data in $L_{w}^{p}\left(\mathbb{R}^{N}\right)$ leading to fast convergence (1.6) is "large", i.e., finite codimensional. We also showed that the basis $\left(e_{n}\right)_{n=1}^{\infty}$ of $L_{w}^{p}\left(\mathbb{R}^{N}\right)$ can be constructed as in a sense arbitrarily small perturbation of any given Schauder basis of $L_{w}^{p}\left(\mathbb{R}^{N}\right)$, if $p>1$.

In this paper we will present how to generalize this result to parabolic equations with more general elliptic parts (1.3), which may have non-constant coefficients and may be of arbitrary order. This will be done in three different versions. The approach in Section 2 is based on the Taylor expansion of the kernel $K$, and the first main result of our paper is formulated in Theorem 2.1. The crucial assumption concerns the interdependence of the space and time variables of the kernel, see (2.1). The second main result, Theorem 3.1, is based on the Fourier-transform, and it is formulated only in the Hilbert-space case $p=2$, although using $L^{p}$-estimates for the Fourier-transform, the proof would generalize to other $p \in(1, \infty)$ as well. The basic assumptions consists of an $L^{2}$-estimate of the derivatives of the kernel and the requirement that the kernel is of convolution type, or slightly more general.

In addition, we will give in Section 4 a 1-d-argument for equations with semigroup kernels with good enough $L^{p}$-estimates of the derivatives, which need not be of convolution type. In the case $N=1$ this result seems to be the most general, although the argument seems not to generalize directly to higher dimensions.

Also, in [1] we only considered initial data in the space $L_{w}^{p}(\mathbb{R})$ with rapidly increasing weights $w$ : here, we also allow weights with polynomial growth rate. (In this case one cannot expect the analogue of (1.6) to hold for all $m$.) In all of the above mentioned cases we present versions with finite polynomial decay rates instead of unlimited $m$ in (1.6). Examples of applications will be discussed in Section 5 .

We remark that our assumptions involve estimates for higher spatial derivatives of the semigroup kernel, which is often a challenging question, but in our opinion not everything possible has been done in the literature yet, and there should be room for future research.

Let us explain some notation. If $N>1$, we use the standard multi-index notation $\alpha=\left(\alpha_{1}, \ldots, \alpha_{N}\right) \in \mathbb{N}_{0}^{N}$ (where $\mathbb{N}_{0}=\{0\} \cup \mathbb{N}$ ) with $|\alpha|=\alpha_{1}+\ldots+\alpha_{N}$ and $\alpha !=\alpha_{1} ! \ldots \alpha_{N} !$. Accordingly, we denote the partial derivatives with respect to the 
variable $x=\left(x_{1}, \ldots, x_{N}\right) \in \mathbb{R}^{N}$ by

$$
D^{\alpha}=D_{x}^{\alpha}=\frac{\partial^{\alpha_{1}}}{\partial x_{1}^{\alpha_{1}}} \ldots \frac{\partial^{\alpha_{N}}}{\partial x_{N}^{\alpha_{N}}}=\partial_{x_{1}}^{\alpha_{1}} \ldots \partial_{x_{N}}^{\alpha_{N}} .
$$

Given $f \in L^{1}\left(\mathbb{R}^{N}\right), N \geq 1$, its Fourier transform is denoted by

$$
\widehat{f}(k)=\mathcal{F} f(k)=\frac{1}{(2 \pi)^{N / 2}} \int_{\mathbb{R}^{N}} e^{-i k \cdot x} f(x) d x, \quad k \in \mathbb{R}^{N},
$$

where $k \cdot x=k_{1} x_{1}+\ldots+k_{N} x_{N}$. We put $x^{2}=x_{1}^{2}+\ldots+x_{N}^{2}$.

Given $p \in[1, \infty]$ we use standard notation $L^{p}\left(\mathbb{R}^{N}\right), L^{p}(0,1)$ etc. for unweighted Lebesgue spaces, the norms of which are denoted by $\|\cdot\|_{p}$. If $p<\infty$ and $w: \mathbb{R}^{N} \rightarrow$ $(0, \infty)$ is a continuous weight function, we denote by $L_{w}^{p}\left(\mathbb{R}^{N}\right)$ the weighted $L^{p}$-space on $\mathbb{R}^{N}$ endowed with the norm

$$
\|f\|_{p, w}:=\left(\int_{\mathbb{R}^{N}}|f(x)|^{p} w(x) d x\right)^{1 / p} .
$$

Given $M \in \mathbb{N}$ (respectively, $M=\infty$ ), we let $\mathcal{B}^{M}\left(\mathbb{R}^{N}\right)$ be the space of all $M$ times continuously differentiable (resp. infinitely smooth) functions $h: \mathbb{R}^{N} \rightarrow \mathbb{R}$ such that $h$ and all partial derivatives of $D^{\alpha} h$ with $|\alpha| \leq M$ (resp. $\alpha \in \mathbb{N}^{N}$ ) exist and are bounded. For example, $h(x)=e^{-x^{2}} \in \mathcal{B}^{\infty}\left(\mathbb{R}^{N}\right)$. If $m \in \mathbb{N}$, we denote by $H^{m}\left(\mathbb{R}^{N}\right)$ the Sobolev-Hilbert-space of functions $f \in L^{2}\left(\mathbb{R}^{N}\right)$ such that the weak partial derivatives $D^{\alpha} f$ belong to $L^{2}\left(\mathbb{R}^{N}\right)$ for all $|\alpha| \leq m$. For these notions, see [8].

As for other general notation, by $C, C^{\prime}$ etc. we denote generic positive constants, the exact value of which may change from place to place. The possible dependence, say, on a parameter $p$ is indicated as $C_{p}$. By supp $f$ we denote the support of a function $f$ and by $\operatorname{sp}(A)$ the linear span of a subset $A$ of a vector space. Its closure is denoted by $\overline{\operatorname{sp}(A)}$. We write $\mathbb{R}^{ \pm}=\{x \in \mathbb{R}: \pm x \geq 0\}$. The characteristic or indicator function of a set $A$ is denoted by $\mathbf{1}_{A}$.

If $X$ denotes a Banach space over the scalar field $\mathbb{K}$ (either $\mathbb{R}$ or $\mathbb{C}$ ), $X^{*}$ stands for its dual. The norm of $X^{*}$ is denoted $\|\cdot\|_{X^{*}}$. The identity operator $X \rightarrow X$ is denoted by $\mathrm{id}_{X}$. For a linear operator $T$ between Banach spaces, $\|T\|$ denotes the operator norm. If $X$ is separable, we recall that a sequence $\left(e_{n}\right)_{n=1}^{\infty} \subset X$ is a Schauder basis (briefly: basis), if every element $f \in X$ can be presented as a convergent sum $f=\sum_{n=1}^{\infty} f_{n} e_{n}$ where the numbers $f_{n} \in \mathbb{K}$ are unique for $f$. An orthonormal basis of a separable Hilbert space is an example. For the general theory of Schauder bases we refer to [5], [9].

Given a basis $\left(e_{n}\right)_{n=1}^{\infty}$ of a separable Banach space $X$ we denote for every $n \in \mathbb{N}$ by $P_{n}$ the basis projection

$$
P_{n} f=P_{n}\left(\sum_{k=1}^{\infty} f_{k} e_{k}\right)=\sum_{k=1}^{n} f_{k} e_{k}, \quad \text { where } f=\sum_{k=1}^{\infty} f_{k} e_{k} \in X .
$$

The number $K=\sup _{n}\left\|P_{n}\right\|$ is called the basis constant of $\left(e_{n}\right)_{n=1}^{\infty}$; the supremum defining $K$ is always finite, see [5]. In [1] we introduced the following notion.

Definition 1.1. Let $x^{*} \in X^{*}$. A Schauder basis $\left(e_{n}\right)_{n=1}^{\infty}$ of $X$ is called shrinking with respect to $x^{*}$, if $\lim _{n \rightarrow \infty}\left\|x^{*} \circ\left(\mathrm{id}_{X}-P_{n}\right)\right\|_{X^{*}}=0$.

For a basis $\left(e_{n}\right)_{n=1}^{\infty}$ of $X$, the biorthogonal functionals $e_{n}^{*} \in X^{*}$ are defined such that $e_{n}^{*}\left(e_{m}\right)=\delta_{m n}$ (Kronecker delta). Denoting $W=\overline{\operatorname{sp}\left\{e_{n}^{*}: n \in \mathbb{N}\right\}} \subset X^{*}$, it is 
easily seen that $\left(e_{n}^{*}\right)_{n=1}^{\infty}$ is a Schauder basis of $W$ with the basis projections $P_{n}^{*}$, where $P_{n}^{*}\left(x^{*}\right)=x^{*} \circ P_{n}$ for $x^{*} \in X^{*}$. However, in general $W \neq X^{*}$. We obtain that $\left(e_{n}\right)_{n=1}^{\infty}$ is shrinking with respect to $x^{*} \in X^{*}$, if and only if $x^{*} \in W$. According to [1], in a reflexive Banach space every basis is shrinking for all bounded functionals.

We will need the following result, which is Theorem 2.2 of [1].

Theorem 1.2. Let $X$ be a separable Banach space, let $x_{m}^{*} \in X^{*}$ for all $m \in \mathbb{N}$, assume that $\left(\tilde{e}_{n}\right)_{n=1}^{\infty}$ is a Schauder basis of $X$ which is shrinking with respect to all $x_{m}^{*}$, and let $\varepsilon>0$ be arbitrary. Then, there exists an increasing sequence $\left(n_{m}\right)_{m=1}^{\infty} \subset$ $\mathbb{N}$ and a basis $\left(e_{n}\right)_{n=1}^{\infty}$ of $X$ such that

$$
x_{m}^{*}\left(e_{n}\right)=0 \text { for all } n \geq n_{m} .
$$

If $T: X \rightarrow X$ is the linear operator with $T \tilde{e}_{n}=e_{n}$ for all $n$, then we have

$$
\left\|\operatorname{id}_{X}-T\right\|<\varepsilon \text {. }
$$

We remark that the functionals $x_{m}^{*} \in X^{*}$ need not be different from each other. We will not use property (1.9) in the proofs of our result, but it yields the additional information that the desired basis can be obtained as a perturbation of any given Schauder basis, in particular, any orthonormal basis.

\section{Approach via Taylor expansion of the Kernel $K$.}

In order to formulate and prove our first main result, we start by describing our assumptions on the semigroup kernel $K$. We now fix $M \in \mathbb{N}$ or $M=\infty$, and assume that the semigroup generated by the operator $A$ has an $M$ times continuously differentiable kernel (1.5) of the form

$$
K(x, y, t)=\frac{d}{t^{b}} h\left((x-y) t^{-a}\right)
$$

for some $h \in \mathcal{B}^{M}\left(\mathbb{R}^{N}\right)$, constants $a, d>0, b \geq 0, x, y \in \mathbb{R}^{N}, t>0$. Clearly, the Gaussian heat kernel of the Laplacian $A=\Delta$ corresponds to the case $a=1 / 2$, $b=N / 2, M=\infty$. More generally, we also consider kernels

$$
K(x, y, t)=\sum_{j=1}^{J} U_{j}(x, t) v_{j}(y) h_{j}\left((x-y) t^{-a_{j}}\right), \quad x \in \mathbb{R}^{N}, t>0,
$$

where $J \in \mathbb{N}$ and, for all $j$, the numbers $a_{j}>0$ are constants, and $v_{j}$ is a bounded and continuous function on $\mathbb{R}^{N}$, and $h_{j} \in \mathcal{B}^{M}\left(\mathbb{R}^{N}\right)$; finally, the measurable functions $U_{j}$ are assumed to satisfy for some constants $C_{j}>0, b_{j} \geq 0$,

$$
U(\cdot, t) \in L^{\infty}\left(\mathbb{R}^{N}\right) \text { for } t>0, \quad\left|U_{j}(x, t)\right| \leq \frac{C_{j}}{t^{b_{j}}} \text { for } x \in \mathbb{R}^{N}, t \geq 1 .
$$

Let $1 \leq p<\infty$. We need to fix a parameter $L \in(0, \infty]$ such that

$$
L>M p+N(p-1),
$$

if $M<\infty$, and $L=\infty$ in the other case. Then, let $w_{L}: \mathbb{R}^{N} \rightarrow \mathbb{R}^{+}$be a continuous weight function satisfying the growth condition

$$
\sup _{x \in \mathbb{R}^{N}} \frac{1}{w_{L}(x)}(1+|x|)^{m}<\infty \quad \forall m \in\{1, \ldots, L\},
$$

where " $m \in\{1, \ldots, L\}$ " is to be read as " $m \in \mathbb{N}$ ", if $L=\infty$.

Assuming these conditions on $K$ and $w$ we state the following result. 
Theorem 2.1. Let first $M=\infty$. There exists a basis $\left(e_{n}\right)_{n=1}^{\infty}$ of the Banach space $L_{w_{L}}^{p}\left(\mathbb{R}^{N}\right)$ and an increasing sequence $\left(n_{m}\right)_{m=1}^{\infty} \subset \mathbb{N}$ with the following property: given $m \in \mathbb{N}$ and initial data

$$
f=\sum_{n=1}^{\infty} f_{n} e_{n} \in L_{w_{L}}^{p}\left(\mathbb{R}^{N}\right),
$$

with $f_{n}=0$ for all $n=1, \ldots, n_{m}$, the solution of (1.1)-(1.2) has the fast decay property

$$
\left\|e^{t A} f\right\|_{\infty} \leq \frac{C_{m, p}}{t^{m}}\|f\|_{p, w_{L}} \text { for all } t \geq 1
$$

If $M<\infty$, then there exists a basis $\left(e_{n}\right)_{n=1}^{\infty}$ of $L_{w_{L}}^{p}\left(\mathbb{R}^{N}\right)$ and a number $n_{M} \in \mathbb{N}$ such that

$$
\left\|e^{t A} f\right\|_{\infty} \leq \frac{C_{M, p}}{t^{a}}\|f\|_{p, w_{L}} \text { for all } t \geq 1
$$

where

$$
a=\min \left\{M a_{j}+b_{j}: j=1, \ldots, J\right\}
$$

for all $f \in \overline{\mathrm{sp}}\left\{e_{n}: n \geq n_{M}\right\}$ (see (2.2), (2.3) for the notation).

The proof of Theorem 2.1 is a generalization of that in [1]. We need to recall a result, which is contained in Theorem 3.1 of the citation. Given a measurable function $h$ on $\mathbb{R}^{N}$ we define in $L_{w_{L}}^{p}\left(\mathbb{R}^{N}\right)$ the functional

$$
\Phi_{h}(f):=\int_{\mathbb{R}^{N}} h(y) f(y) d y,
$$

if the integral converges for all $f \in L_{w_{L}}^{p}\left(\mathbb{R}^{N}\right)$.

Theorem 2.2. Let $1 \leq p<\infty$ and let for all $m \in \mathbb{N}$ the functions $h_{m}: \mathbb{R}^{N} \rightarrow \mathbb{R}$ be measurable such that, if $p>1$,

$$
\int_{\mathbb{R}^{N}}\left(\frac{\left|h_{m}(y)\right|^{p}}{w_{L}(y)}\right)^{1 /(p-1)} d y<\infty,
$$

or, if $p=1$,

$$
\begin{aligned}
& \text { all } h_{m} / w_{L} \text { are continuous and can be continuously } \\
& \text { extended to }[-\infty, \infty]^{N} \text {. }
\end{aligned}
$$

Then, every $\Phi_{h_{m}}$ as in (2.10) is a bounded linear functional on $L_{w_{L}}^{p}\left(\mathbb{R}^{N}\right)$, and there are a Schauder basis $\left(e_{n}\right)_{n=1}^{\infty}$ of $L_{w_{L}}^{p}\left(\mathbb{R}^{N}\right)$ and indices $0<n_{1}<n_{2}<\ldots$ such that $\Phi_{h_{m}}\left(e_{n}\right)=0$ for all $n \geq n_{m}$.

Here, the metric space $[-\infty, \infty]^{N}$ is defined as the $N$-fold Cartesian product of the two-point compactification of $(-\infty, \infty)$. For the proof of Theorem 2.2 , see the above mentioned reference.

Proof of Theorem 2.1. We consider only the general case (2.2) instead of (2.1). Fix $x \in \mathbb{R}^{N}, t>0$ and for all $j=1, \ldots, J$, put

$$
h_{j, x}(y)=h_{j}\left((x-y) t^{-a_{j}}\right), \quad y \in \mathbb{R}^{N} .
$$


For any $\alpha \in \mathbb{N}_{0}^{N}$ we have

$$
D^{\alpha} h_{j, x}(y)=\frac{(-1)^{|\alpha|}}{t^{a_{j}|\alpha|}}\left(D^{\alpha} h_{j}\right)\left((x-y) t^{-a_{j}}\right) .
$$

Let us denote by $m(j, \alpha)$ an ordering of all pairs $(j, \alpha)$, where $j=1, \ldots, J$ and $\alpha \in \mathbb{N}_{0}$, such that in particular $m(j, \alpha)<m(k, \beta)$ for all $\alpha, \beta$ with $|\alpha|<|\beta|$. Then, if $M=\infty$, we define for every $m \in \mathbb{N}_{0}$ the functions $h_{m}$,

$$
h_{m}(y)=v_{j}(y) y^{\alpha}, \quad y \in \mathbb{R}^{N},
$$

where $(j, \alpha)$ is such that $m=m(j, \alpha)$. If $M<\infty$, we define $h_{m}$ by (2.15), if $m=m(j, \alpha)$ with $|\alpha|<\widetilde{m}=M$, and $h_{m}(y) \equiv 1$ for all other $m$; this last definition does not play any important role later, see also the remark just after Theorem 1.2.

If $M=\infty$, it follows easily from the choice of the weight in (2.5) and the boundedness and continuity of $v_{j}$, that the functions $h_{m}$ satisfy the assumptions of Theorem 2.2. If $M<\infty$, we obtain from (2.5) the estimate $w_{L}(y) \geq C(1+|y|)^{L}$ and thus (2.15), (2.4) and $p^{\prime}=p /(p-1)$ imply for all $m \leq M, p>1$,

$$
\begin{aligned}
& \int_{\mathbb{R}^{N}}\left(\frac{\left|h_{m}(y)\right|^{p}}{w_{L}(y)}\right)^{1 /(p-1)} d y \leq C_{M} \int_{\mathbb{R}^{N}}|y|^{|\alpha| p^{\prime}}(1+|y|)^{-L /(p-1)} d y \\
\leq & C_{M} \int_{\mathbb{R}^{N}}(1+|y|)^{M p^{\prime}-L /(p-1)} d y \leq C_{M} \int_{\mathbb{R}^{N}}(1+|y|)^{-N-\delta} d y<\infty
\end{aligned}
$$

for some constant $\delta>0$. If $p=1$, we obtain for all $m \leq M<L$ from (2.5) that $\left|h_{m}(y) / w_{L}(y)\right| \rightarrow 0$ as $|y| \rightarrow \infty$ so that (2.12) holds by setting $h_{m} / w_{L}=0$ on the boundary of $(-\infty, \infty)^{N}$. Hence, the assumptions of Theorem 2.2 hold also in this case (the case $m>M$ is trivial since $h_{m}=1$ there) so that $\Phi_{h_{m}}$ are bounded functionals on $L_{w_{L}}^{p}\left(\mathbb{R}^{N}\right)$ and we find a Schauder basis $\left(e_{n}\right)_{n=1}^{\infty}$ of $L_{w_{L}}^{p}\left(\mathbb{R}^{N}\right)$ and indices $\nu_{1}<\nu_{2}<\ldots$ such that

$$
\Phi_{h_{m}}\left(e_{n}\right)=0 \text { for all } n \geq \nu_{m} .
$$

If $M<\infty$, we define the number $n_{M}$, appearing in the statement (2.8) of the theorem, as follows: if $m(j, \alpha)$ is the largest number under the condition $|\alpha|<M$, then we set

$$
n_{M}:=\nu_{m(\alpha, j)}
$$

This, the choice of the numbers $m(j, \alpha)$ and (2.16) imply that

$$
\Phi_{h_{m(\alpha, j)}}\left(e_{n}\right)=0 \text { for all } n \geq n_{M} \text {, all } \alpha \text { with }|\alpha|<M \text { and all } 1 \leq j \leq J .
$$

In the case $M=\infty$ we take an arbitrary $m \in \mathbb{N}$ and then again define $m(j, \alpha)$ as the largest number such that $|\alpha|<m$. For the number $n_{m}$ in (2.6) we choose $n_{m}:=\nu_{m(\alpha, j)}$. Again we get

$$
\Phi_{h_{m(\alpha, j)}}\left(e_{n}\right)=0 \text { for all } n \geq n_{m} \text {, all } \alpha \text { with }|\alpha|<m \text { and all } 1 \leq j \leq J .
$$

For the rest of the proof we take an arbitrary $m \in \mathbb{N}$, if $M=\infty$, and $m=M$ in the case $M<\infty$. Recall that $h_{j} \in \mathcal{B}^{M}\left(\mathbb{R}^{N}\right)$ for every $j=1, \ldots, J$. Then, the multidimensional Taylor formula yields for all $j$ a function $\bar{y}_{j}(y)$ such that

$$
h_{j, x}(y)=\sum_{|\alpha|<m} \frac{D^{\alpha} h_{j, x}(0)}{\alpha !} y^{\alpha}+\sum_{|\alpha|=m} \frac{D^{\alpha} h_{j, x}\left(\bar{y}_{j}(y)\right)}{\alpha !} y^{\alpha}, \quad y \in \mathbb{R}^{N} .
$$


Hence, by (2.15),

$$
\begin{aligned}
& \sum_{j=1}^{J} U_{j}(x, t) v_{j}(y) h_{j, x}(y) \\
= & \sum_{|\alpha|<m} \sum_{j=1}^{J} U_{j}(x, t) \frac{D^{\alpha} h_{j, x}(0)}{\alpha !} v_{j}(y) y^{\alpha}+\sum_{|\alpha|=m} \sum_{j=1}^{J} U_{j}(x, t) v_{j}(y) \frac{D^{\alpha} h_{j, x}\left(\bar{y}_{j}(y)\right)}{\alpha !} y^{\alpha} \\
= & \sum_{|\alpha|<m} \sum_{j=1}^{J} \frac{U_{j}(x, t) D^{\alpha} h_{j, x}(0)}{\alpha !} h_{m(\alpha, j)}(y) \\
(2.19)+ & \sum_{|\alpha|=m} \sum_{j=1}^{J} U_{j}(x, t) v_{j}(y) \frac{D^{\alpha} h_{j, x}\left(\bar{y}_{j}(y)\right)}{\alpha !} y^{\alpha},
\end{aligned}
$$

where $x, y \in \mathbb{R}^{N}, t>0$. For $\alpha$ with $|\alpha|=m$ we obtain constants $c_{j, \alpha, m}$ (in particular independent of $t$ ), such that

$$
\sup _{x, y}\left|\frac{D^{\alpha} h_{j, x}\left(\bar{y}_{j}(y)\right)}{\alpha !}\right| \leq \frac{c_{j, \alpha, m}}{t^{m a_{j}}}
$$

for all $j, t \geq 1$. This follows from (2.14) and the fact that $h_{j} \in \mathcal{B}^{M}\left(\mathbb{R}^{N}\right)$. Now let $f=\sum_{n \geq n_{m}} f_{n} e_{n} \in L_{w_{L}}^{p}\left(\mathbb{R}^{N}\right)$. We get by $(2.13),(2.19)$,

$$
\begin{aligned}
& \left|e^{t A} f(x)\right|=\left|\sum_{j=1}^{J} U_{j}(x, t) \int_{\mathbb{R}^{N}} v_{j}(y) h_{j, x}(y) f(y) d y\right| \\
= & \mid \sum_{j=1}^{J} \sum_{|\alpha|<m} U_{j}(x, t) \frac{D^{\alpha} h_{j, x}(0)}{\alpha !} \Phi_{h_{m(\alpha, j)}}\left(\sum_{n \geq n_{m}} f_{n} e_{n}\right) \\
& +\sum_{|\alpha|=m} \sum_{j=1}^{J} U_{j}(x, t) \int_{\mathbb{R}^{N}} \frac{D^{\alpha} h_{j, x}(\bar{y}(y))}{\alpha !} v_{j}(y) y^{\alpha} f(y) d y \mid, \quad x \in \mathbb{R}^{N}, t \geq 1 .
\end{aligned}
$$

Here, due to (2.17), only the last line is non-zero, and it can be bounded using (2.3), (2.20) by

$$
\begin{aligned}
& \sum_{|\alpha|=m} \sum_{j=1}^{J}\left|U_{j}(x, t)\right| \int_{\mathbb{R}^{N}}\left|\frac{D^{\alpha} h_{j, x}(\bar{y}(y))}{\alpha !} v_{j}(y) y^{\alpha} f(y)\right| d y \\
\leq & \sum_{|\alpha|=m} \sum_{j=1}^{J} \frac{C_{j, \alpha, m}}{t^{a_{j} m+b_{j}}} \int_{\mathbb{R}^{N}}\left|v_{j}(y) y^{\alpha} f(y)\right| d y \\
\leq & C_{m, p} \frac{1}{t^{a}}\|f\|_{p, w_{L}}
\end{aligned}
$$

where $x \in \mathbb{R}^{N}, t \geq 1, a$ is as in (2.9), and $C_{j, \alpha, m}$ and $C_{m, p}$ are constants. This yields (2.8) and also (2.7) since $m$ was arbitrary in the case $M=\infty$.

\section{Approach Using Fourier anAlysis.}

In this section we describe an alternative approach using the Fourier-transform. The result overlaps with that in Section 2, but there are also some obvious differences 
in the assumptions of Theorems 2.1 and 3.1. We start by fixing an even $M \in \mathbb{N}$ such that $M-N \geq 2$, or $M=\infty$, and assume that the weight $w_{M}: \mathbb{R}^{N} \rightarrow \mathbb{R}^{+}$ is as in (2.5), with $M$ replacing $L$. In the following, if $M=\infty$, the notation $M / 2$ is to be read as $\infty$, and inequalities like $x \leq M$ for some real number $x$ are to be interpreted as no condition.

We assume that the kernel $K(x, y, t),(1.5)$, is of convolution type, $K(x, y, t)=$ $\widetilde{K}(x-y, t)$, and that in addition the function $x \mapsto D_{x}^{\alpha} \widetilde{K}(x, t)$ belongs to $L^{2}\left(\mathbb{R}^{N}\right)$ for every $\alpha \in \mathbb{N}_{0}^{N}$ with $|\alpha| \leq M / 2$ and $t>0$, and the function $t \mapsto \widetilde{K}(\cdot, t)$ is continuous as a map from $(0, \infty)$ to $L^{2}\left(\mathbb{R}^{N}\right)$. See the remark at the end of this section about possible more general forms of the kernel. Note that in (3.1) and later in similar places the differentiation is with respect to the space variable.

Theorem 3.1. Let the weight $w_{M}$ and the kernel $\widetilde{K}$ be as described above, and assume that there exist constants $a>0$ and $b \in \mathbb{R}$ such that the kernel satisfies for all $t \geq 1$ the estimate

$$
\left\|D^{\alpha} \widetilde{K}(\cdot, t)\right\|_{2} \leq \frac{C}{t^{a|\alpha|+b}}
$$

for all multi-indices $\alpha$ with $|\alpha| \leq \mu$, where $\mu \in \mathbb{N}$ is the largest integer not bigger than $M / 2-N / 2$.

If $M=\infty$, there exists a basis $\left(e_{n}\right)_{n=1}^{\infty}$ of the Banach space $L_{w_{M}}^{2}\left(\mathbb{R}^{N}\right)$ and an increasing sequence $\left(n_{m}\right)_{m=1}^{\infty}$ with the following property: given $m \in \mathbb{N}$, then for any initial data

$$
f=\sum_{n=n_{m}}^{\infty} f_{n} e_{n} \in L_{w_{M}}^{2}\left(\mathbb{R}^{N}\right),
$$

the solution of (1.1)-(1.2) has the bound

$$
\left\|e^{t A} f\right\|_{\infty} \leq \frac{C_{m}}{t^{m}}\|f\|_{2, w_{M}} \text { for all } t \geq 1
$$

If $M<\infty$, then there exists a basis $\left(e_{n}\right)_{n=1}^{\infty}$ of $L_{w_{M}}^{2}\left(\mathbb{R}^{N}\right)$ and a number $n_{M} \in \mathbb{N}$ such that

$$
\left\|e^{t A} f\right\|_{\infty} \leq \frac{C_{M}}{t^{\mu a+b}}\|f\|_{2, w_{M}} \text { for all } t \geq 1
$$

for all $f \in \overline{\operatorname{sp}}\left\{e_{n}: n \geq n_{M}\right\}$.

We aim to use Theorem 1.2 and work with the Fourier transform. For all multiindices $\alpha$ with $|\alpha| \leq M / 2$ we define the linear functionals by

$$
J^{\alpha} f=\int_{\mathbb{R}^{N}} y^{\alpha} f(y) d y .
$$

If $M<\infty$, we define in addition for example $J^{\alpha} f:=\int_{\mathbb{R}^{N}} f(y) d y$ for all $\alpha$ with $|\alpha|>$ $M / 2$. Due to the choice of the weight $w_{M}$, these functionals are bounded mappings $L_{w_{M}}^{2}(\mathbb{R}) \rightarrow \mathbb{C}$. Then, again due to the choice of $w_{M}$, we have $x^{\alpha} f \in L^{2}\left(\mathbb{R}^{N}\right)$ for all $|\alpha| \leq M / 2$ and there holds

$$
D^{\alpha} \widehat{f}(k)=(-i)^{|\alpha|} \mathcal{F}\left(x^{\alpha} f(x)\right)(k), \quad k \in \mathbb{R}^{N},
$$


at least for the weak partial derivatives. Thus, the Fourier transform $\widehat{f}$ belongs to the Sobolev space $H^{M / 2}\left(\mathbb{R}^{N}\right)$ for every $f \in L_{w_{M}}^{2}\left(\mathbb{R}^{N}\right)$, and we have

$$
D^{\alpha} \widehat{f} \in L^{2}\left(\mathbb{R}^{N}\right), \quad\left\|D^{\alpha} \widehat{f}\right\|_{2} \leq C_{\alpha}\|f\|_{2, w_{M}} \text { for all }|\alpha| \leq M / 2 .
$$

By the Sobolev embedding theorem, see for example [8], Theorem 7.25, every function $g \in H^{M / 2}\left(\mathbb{R}^{N}\right)$ is at least $\mu$ times continuously differentiable, where $\mu$ is as in (3.1), so that we obtain for every multi-index $\beta$ with $|\beta| \leq \mu$ and every compact set $B \subset \mathbb{R}^{N}$,

$$
\sup _{k \in B}\left|D^{\beta} g(k)\right| \leq C_{B} \max _{|\alpha| \leq M / 2}\left\|D^{\alpha} g\right\|_{2},
$$

where the expression on the right is equivalent to the norm of the Sobolev space $H^{M / 2}\left(\mathbb{R}^{N}\right)$. Combining this with (3.7) we obtain

$$
\sup _{k \in B}\left|D^{\alpha} \widehat{f}(k)\right| \leq C_{B}\|f\|_{2, w_{M}}
$$

for every compact set $B \subset \mathbb{R}^{N}$, function $f \in L_{w_{M}}^{2}\left(\mathbb{R}^{N}\right)$, and index $|\alpha| \leq \mu$.

Lemma 3.2. If $f \in L_{w_{M}}^{2}\left(\mathbb{R}^{N}\right)$ and $J^{\alpha} f=0$ for all multi-indices $\alpha$ with $|\alpha| \leq \mu-1$, then all partial derivatives $D^{\alpha} \widehat{f}$ with $|\alpha| \leq \mu-1$ vanish at 0 . Consequently, we can write

$$
\widehat{f}(k)=\sum_{|\alpha|=\mu} k^{\alpha} g_{\alpha}(k)
$$

where $g_{\alpha} \in L^{2}\left(\mathbb{R}^{N}\right) \cap C\left(\mathbb{R}^{N}\right)$, and

$$
\left\|g_{\alpha}\right\|_{2} \leq C_{\alpha}\|f\|_{2, w_{M}},
$$

Proof. Let us denote by $\mathbb{B}_{N}$ the closed unit ball of $\mathbb{R}^{N}$.

The vanishing of the derivatives $D^{\alpha} \widehat{f}(0)$ for all $|\alpha| \leq \mu-1$ is a direct consequence of the relations (3.6), the assumption on the functionals $J^{\alpha}$, and

$$
\mathcal{F}\left(x^{\alpha} f\right)(0)=\frac{1}{(2 \pi)^{N / 2}} \int_{\mathbb{R}^{N}} e^{i \overline{0} \cdot x} x^{\alpha} f(x) d x=\frac{1}{(2 \pi)^{N / 2}} J^{\alpha} f .
$$

Then, one obtains the formula

$$
\widehat{f}(k)=\sum_{|\alpha|=\mu} k^{\alpha} \check{g}_{\alpha}(k)
$$

from the Taylor expansion of $\mu$ th degree for $\widehat{f}$ at 0 (cf. (2.18)); here every function $\check{g}_{\alpha}$ is continuous, since $\widehat{f} \in C^{\mu}\left(\mathbb{R}^{N}\right)$. Moreover, for $k \in 2 \mathbb{B}_{N}=\{k:|k| \leq 2\}$ we have $\check{g}_{\alpha}(k)=D^{\alpha} \widehat{f}(\kappa(k))$, where $\kappa$ is a continuous function with values in $2 \mathbb{B}_{N} \subset \mathbb{R}^{N}$, so that we can bound by using (3.8)

$$
\sup _{k \in 2 \mathbb{B}_{N}}\left|\check{g}_{\alpha}(k)\right| \leq C \sup _{k \in 2 \mathbb{B}_{N}}\left|D^{\alpha} \widehat{f}\right| \leq C^{\prime}\|f\|_{2, w_{M}} .
$$

To treat the domain $\mathbb{R}^{N} \backslash \mathbb{B}_{N} \ni k$, one defines an open covering of the surface $\mathbb{S}_{N}=\left\{k \in \mathbb{R}^{N}:|k|=1\right\}$ by some sets $\Omega_{\alpha} \subset \mathbb{S}_{N}$, indexed by multi-indices $\alpha$ with $|\alpha|=\mu$, such that $\left|k^{\alpha}\right| \geq 1 / N^{2}$ for all $k \in \Omega_{\alpha}$, and then defines a smooth partition 
of unity $\left(\chi_{\alpha}\right)_{|\alpha|=\mu}$, where $\chi_{\alpha}: \mathbb{S}_{N} \rightarrow[0,1]$ for all $\alpha$, subordinate to this covering. One writes

$$
\widehat{f}(k)=\sum_{|\alpha|=\mu} k^{\alpha} \widetilde{g}_{\alpha}(k)
$$

with $\widetilde{g}_{\alpha}(k)=\chi_{\alpha}(k /|k|) \widehat{f}(k) / k^{\alpha}$. We find that $\widetilde{g}_{\alpha}: \mathbb{R}^{N} \backslash \mathbb{B}_{N} \rightarrow \mathbb{C}^{N}$ is a continuous function whose norm in the space $L^{2}\left(\mathbb{R}^{N} \backslash \mathbb{B}_{N}\right)$ is bounded by a constant times $\|\widehat{f}\|_{2}$.

Finally, one defines a continuous cut-off function $\mathcal{X}: \mathbb{R}^{N} \rightarrow[0,1]$ such that $\mathcal{X}(k)=1$ for $k \in \mathbb{B}_{N}$ and $\mathcal{X}(k)=0$ for $k \in \mathbb{R}^{N} \backslash 2 \mathbb{B}_{N}$. Writing $g_{\alpha}=\mathcal{X}_{g_{\alpha}}+(1-\mathcal{X}) \widetilde{g}_{\alpha}$ we obtain (3.9) $-(3.10)$.

Proof of Theorem 3.1. We obtain the desired Schauder basis $\left(e_{n}\right)_{n=1}^{\infty}$ from Theorem 1.2 , where we take the space $L_{w_{M}}^{2}\left(\mathbb{R}^{N}\right)$ for $X$ and the functionals $J^{\alpha}$ for $x_{m}^{*}$. If $M<\infty$, we show that (3.4) holds, if the initial data satisfies

$$
J^{\alpha} f=0
$$

for all $|\alpha| \leq \mu-1$. Then, in the case $M=\infty$ we obtain (3.3), since we can apply the result with finite $M$, where the number $\mu$ can be chosen arbitrarily large.

Let us fix $f$ such that (3.11) holds. Then, Lemma 3.2 applies, and we can write $\widehat{f}$ with the help of the functions $g_{\alpha}$ as in (3.9). By the assumptions on the semigroup, the function $x \mapsto D_{x}^{\alpha} \widetilde{K}(x, t)$ belongs to $L^{2}\left(\mathbb{R}^{N}\right)$ for all $t>0$, hence, by the Plancherel theorem the function

$$
\mathcal{F}\left(D_{x}^{\alpha} \widetilde{K}(x, t)\right)
$$

is also in $L^{2}\left(\mathbb{R}^{N}\right)$; here and later, we consider the Fourier-transform with respect to the $x$-variable. Moreover, due to (3.1) and the Plancherel theorem we have for $|\alpha| \leq \mu$ and $t>0$ the estimate

$$
\left\|\mathcal{F}\left(D_{x}^{\alpha} \widetilde{K}(x, t)\right)\right\|_{2} \leq \frac{C}{(t+1)^{a|\alpha|+b}} .
$$

By the Young inequality, $f \in L^{1}\left(\mathbb{R}^{N}\right)$ and $\widetilde{K}(\cdot, t) \in L^{2}\left(\mathbb{R}^{N}\right)$ imply

$$
\widetilde{K}(\cdot, t) * f(x)=\int_{\mathbb{R}^{N}} \widetilde{K}(x-y) f(y) d y \in L^{2}\left(\mathbb{R}^{N}\right)
$$

and also the formula for the commutation of the Fourier transform and convolution ([8], Theorem 7.19) generalizes to this case, so that we get

$$
\begin{aligned}
e^{t A} f(x) & =\widetilde{K}(\cdot, t) * f(x)=\mathcal{F}^{-1} \mathcal{F}(\widetilde{K}(\cdot, t) * f)(x) \\
& =(2 \pi)^{-N / 2} \int_{\mathbb{R}^{N}} e^{i x k}(\mathcal{F} \widetilde{K}(\cdot, t))(k) \widehat{f}(k) d k \\
& =(2 \pi)^{-N / 2} \sum_{|\alpha|=\mu} \int_{\mathbb{R}^{N}} e^{i x k} \mathcal{F}(\widetilde{K}(\cdot, t))(k) k^{\alpha} g_{\alpha}(k) d k \\
& =(2 \pi)^{-N / 2} \sum_{|\alpha|=\mu}(-i)^{-|\alpha|} \int_{\mathbb{R}^{N}} e^{i x k} \mathcal{F}\left(D_{x}^{\alpha} \widetilde{K}(x, t)\right)(k) g_{\alpha}(k) d k,
\end{aligned}
$$


where we also used

$$
\mathcal{F}\left(D_{x}^{\alpha} \widetilde{K}(x, t)\right)(k)=(-i)^{|\alpha|} k^{\alpha} \mathcal{F}(\widetilde{K}(\cdot, t))(k)
$$

Hence, according to (3.12), (3.10) and the Cauchy-Schwartz inequality

$$
\begin{aligned}
\left|e^{t A} f(x)\right| & \leq \sum_{|\alpha|=\mu} \int_{\mathbb{R}^{N}} \mid \mathcal{F}\left(D_{x}^{\alpha} \widetilde{K}(x, t)|| g_{\alpha}(k) \mid d k\right. \\
& \leq \sum_{|\alpha|=\mu}\left\|\mathcal{F}\left(D^{\alpha} \widetilde{K}(\cdot, t)\right)\right\|_{2}\left\|g_{\alpha}\right\|_{2} \leq \frac{C}{(1+t)^{a \mu+b}}\|f\|_{2, w_{M}} .
\end{aligned}
$$

We finally remark that the above approach also works for more general kernels

$$
K^{\prime}(x, y, t)=U(x, t) \widetilde{K}(x-y, t),
$$

where $\widetilde{K}$ is as in Theorem 3.1 and $U$ is a measurable function $\mathbb{R}^{N} \times(0, \infty)$ such that for some constants $C>0, \beta \in \mathbb{R}$,

$$
U(\cdot, t) \in L^{\infty}\left(\mathbb{R}^{N}\right) \text { for } t>0, \quad|U(x, t)| \leq \frac{C}{t^{\beta}} \text { for } x \in \mathbb{R}^{N}, t \geq 1 .
$$

We obtain the result of Theorem 3.1, where the exponent $\mu a+b$ in (3.4) is replaced by $\mu a+b+\beta$. As for the proof, we just write

$$
e^{t A} f(x)=U(x, t) \widetilde{K}(\cdot, t) * f
$$

and apply (3.14)-(3.15) to estimate the convolution.

\section{Approach Using REPEATED integration FUnCtionals.}

In this section we describe yet another approach which allows us to relax the specific assumptions on the form of the $x$ - and $y$-dependence of the semigroup kernel $K$ in Theorems 2.1 and 3.1. However, our proof only works in one space dimension so that we fix $N=1$ for this section. As for the operator $A$ in equation (1.1) and its associated semigroup kernel $K,(1.5)$, we assume that there exist constants $a>0$ and $b \in \mathbb{R}$ such that, for some index $M \in \mathbb{R} \cup\{\infty\}$,

$$
\left|\partial_{y}^{m} K(x, y, t)\right| \leq \frac{C}{(t+1)^{a m+b}}
$$

for all $m \leq M$ (for all $m \in \mathbb{N}$, if $M=\infty$ ) and all $x, y \in \mathbb{R}, t>0$.

In order to formulate the main result of this section, we select the space of initial data to be $L_{w_{Q}}^{p}(\mathbb{R})$, where $1 \leq p<\infty$ and we fix $Q$ such that

$$
Q>p(M+1)+1
$$

if $M<\infty$, and $Q=\infty$ in the other case. The weight $w_{Q}: \mathbb{R}^{N} \rightarrow \mathbb{R}^{+}$is as in (2.5), with $Q$ replacing $L$.

Theorem 4.1. If $M=\infty$, there exists a basis $\left(e_{n}\right)_{n=1}^{\infty}$ of the Banach space $L_{w_{Q}}^{p}(\mathbb{R})$ and an increasing sequence $\left(n_{m}\right)_{m=1}^{\infty}$ with the following property: given $m \in \mathbb{N}$, then for any initial data

$$
f=\sum_{n=n_{m}}^{\infty} f_{n} e_{n} \in L_{w_{Q}}^{p}(\mathbb{R})
$$


the solution of (1.1)-(1.2) has the estimate

$$
\left\|e^{t A} f\right\|_{\infty} \leq \frac{C_{m}}{t^{m}}\|f\|_{p, w_{Q}} \text { for all } t \geq 1 \text {. }
$$

If $M<\infty$, then there exists a basis $\left(e_{n}\right)_{n=1}^{\infty}$ of $L_{w_{Q}}^{p}(\mathbb{R})$ and a number $n_{M} \in \mathbb{N}$ such that

$$
\left\|e^{t A} f\right\|_{\infty} \leq \frac{C_{M}}{t^{M a+b}}\|f\|_{p, w_{Q}} \text { for all } t \geq 1
$$

for all $f \in \operatorname{sp}\left\{e_{n}: n \geq n_{M}\right\}$.

First, for all $m \leq M$ (for all $m \in \mathbb{N}$, if $M=\infty$ ) we define the linear operator of repeated integrations and the corresponding functional as follows:

$$
\begin{aligned}
I^{m} f(x) & =\frac{1}{(m-1) !} \int_{-\infty}^{x}(x-y)^{m-1} f(y) d y, \\
J^{m} f & =\frac{1}{(m-1) !} \int_{-\infty}^{0}(-y)^{m-1} f(y) d y, \quad m \in \mathbb{N} .
\end{aligned}
$$

Notice that indeed

$$
I^{m} f(x)=\int_{-\infty}^{x} I^{m-1} f(y) d y \text { and } \quad J^{m} f=\int_{-\infty}^{0} I^{m-1} f(y) d y
$$

for all $m \geq 2$. If $M<\infty$, we set $J^{m} f=\int_{\mathbb{R}} f(y) d y$ for $m>M$. We denote $L_{w_{Q}}^{p,-}(\mathbb{R}):=\left\{f \in L_{w_{Q}}^{p}(\mathbb{R}): \operatorname{supp} f \subset \mathbb{R}^{-}\right\}$.

Lemma 4.2. ( $i)$ There exists $\delta>0$ such that if $m \leq M($ or $m \in \mathbb{N}$, if $M=\infty$ ) and $f \in L_{w_{Q}}^{p}(\mathbb{R})$, then the restriction of $I^{m} f$ to $\mathbb{R}^{-}$satisfies

$$
\sup _{x \in \mathbb{R}^{-}}(1+|x|)^{1+\delta}\left|I^{m} f(x)\right| \leq C_{M, p}\|f\|_{p, w_{Q}}<\infty .
$$

(ii) If $m \leq M$ (or $m \in \mathbb{N}$, if $M=\infty$ ) is given and $f \in L_{w_{Q}}^{p,-}(\mathbb{R})$ has the property that $J^{k} f=0$ for all $k \in \mathbb{N}$ with $k \leq m$, then

$$
\operatorname{supp} I^{k} f \subset \mathbb{R}^{-}
$$

for all $k \leq m$. In particular $I^{k} f \in L^{1}(\mathbb{R})$ and

$$
\left\|I^{k} f\right\|_{1} \leq C_{k, p, w_{Q}}\|f\|_{p, w_{Q}}
$$

for every $k \leq m$.

(iii) Every $\bar{J}^{m}$ is a bounded linear functional on $L_{w_{Q}}^{p}(\mathbb{R})$.

Proof. As for $(i)$, we denote the dual exponent $p^{\prime}=p /(p-1)$ and choose

$$
\delta:=\frac{Q-p(M+1)-1}{2 p-1}>0,
$$

if $M$ is finite, or any $\delta>0$, if $M=\infty$. Then, (4.6) and the Hölder inequality imply for $x \leq 0$

$$
(1+|x|)^{1+\delta}\left|I^{m} f(x)\right| \leq C_{m}(1+|x|)^{1+\delta} \int_{-\infty}^{x}|x-y|^{m-1}|f(y)| d y
$$




$$
\begin{aligned}
& \leq C_{m}(1+|x|)^{1+\delta} \int_{-\infty}^{x}|y|^{m-1}(1+|y|)^{-\delta-m-\frac{1+\delta}{p^{\prime}}}(1+|y|)^{\delta+m+\frac{1+\delta}{p^{\prime}}}|f(y)| d y \\
& \leq C_{m} \int_{-\infty}^{x}(1+|y|)^{-\frac{1+\delta}{p^{\prime}}}(1+|y|)^{\delta+m+\frac{1+\delta}{p^{\prime}}}|f(y)| d y \\
& \leq C_{m}\left(\int_{-\infty}^{0}(1+|y|)^{-1-\delta} d y\right)^{1 / p^{\prime}}\left(\int_{-\infty}^{0}(1+|y|)^{p m+\frac{p}{p^{\prime}}+\delta\left(p+\frac{p}{p^{\prime}}\right)}|f(y)|^{p} d y\right)^{1 / p} \\
& \leq C_{k, m, p}\left(\int_{-\infty}^{0} w_{Q}(y)|f(y)|^{p} d y\right)^{1 / p} \leq C_{k, m, p}\|f\|_{p, w_{Q}} \cdot
\end{aligned}
$$

Here, in the case of finite $Q$, we used

$$
p m+\frac{p}{p^{\prime}}+\delta\left(p+\frac{p}{p^{\prime}}\right)=p(m+1)-1+\delta(2 p-1) \leq Q .
$$

The proof for the case $p=1$ is simpler.

Concerning (ii), a straightforward induction argument yields (4.9): assume that $J^{k} f=0$ for all $k \leq m$ and that $\tilde{m}<m$ and (4.9) holds for all $k \leq \tilde{m}$. Then, by the definition of $I^{\tilde{m}+1}$, for $x \geq 0$,

$$
I^{\tilde{m}+1} f(x)=\int_{-\infty}^{0} I^{\tilde{m}} f(y) d y+\int_{0}^{x} I^{\tilde{m}} f(y) d y
$$

Here, the first term equals $J^{\tilde{m}+1} f$ and is thus 0 , and the second term also vanishes by the induction assumption. The bound (4.10) follows from (4.9), (4.8) and an application of the Hölder inequality.

The statement $(i i i)$ is a consequence of the Hölder inequality and the choice of the number $Q$ and the weight $w_{Q}$.

The next result about decay rates can now be obtained by a simple integration by parts argument.

Proposition 4.3. Let $N=1$, assume (4.1) holds for the kernel $K$, and let $f \in$ $L_{w_{Q}}^{p,-}(\mathbb{R})$ be such that $J^{m} f=0$ for all $m \in \mathbb{N}$ with $m \leq M$. Then, there holds the bound

$$
\left\|e^{t A} f\right\|_{\infty} \leq \frac{C_{p, M}\|f\|_{p, w_{Q}}}{t^{a M}}, \quad t \geq 1
$$

Proof. We employ repeated integration by parts $M$ times with respect to $y$ in order to evaluate (1.5):

$$
u(x, t)=\int_{\mathbb{R}}\left(\partial_{y}^{M} K(x, y, t)\right)\left(I^{M} f\right)(y) d y .
$$

The replacement terms vanish, since by Lemma 4.2.(i), (ii), for all $m \leq M$ the function $I^{m} f(y)$ decays to 0 as $y \rightarrow \pm \infty$ and also $\partial_{y}^{m} K(x, y, t)$ is a bounded function 
of $y$. The bound (4.11) for $t \geq 1$ follows from (4.1) and (4.8):

$$
\begin{aligned}
& \left|\int_{\mathbb{R}}\left(\partial_{y}^{M} K(x, y, t)\right)\left(I^{M} f\right)(y) d y\right| \leq \sup _{x, y \in \mathbb{R}}\left|\partial_{y}^{M} K(x, y, t)\right| \int_{\mathbb{R}}\left|\left(I^{M} f\right)(y)\right| d y \\
\leq & \frac{C_{p, M}}{t^{a M+b}}\|f\|_{p, w_{Q}} \cdot \square
\end{aligned}
$$

The authors do not know a straightforward generalization of this argument to higher dimensional spaces $\mathbb{R}^{N} \ni x$. Problems are caused by the more complicated replacement terms with mixtures of definite and indefinite integrals.

Proof of Theorem 4.1. Let $p$ and $w$ be as in the assumption and first consider the Banach space $X=L_{w}^{p,-}(\mathbb{R})$. The functionals $J^{m}=: x_{m}^{*}$ of $(4.7)$ are well defined and bounded on $X$, by Lemma $4.2,(i i i)$. We fix a basis $\left(\tilde{e}_{n}^{-}\right)_{n=1}^{\infty}$, which is shrinking with respect to all $x_{m}^{*}$; in the case $p=1$ we use the proof of Theorem 2.2 to find this. Then, Theorem 1.2 yields the desired basis $\left(e_{n}^{-}\right)_{n=1}^{\infty}$ of $L_{w}^{p,-}(\mathbb{R})$ and the sequence of indices $\left(n_{m}\right)_{m=1}^{\infty}$; in particular, given $m \in \mathbb{N}$ we have

$$
J^{k}\left(e_{n}^{-}\right)=0
$$

for every $k \leq m, n \geq n_{m}$. To see that (4.4) holds for a given $m$ and for any initial data $f^{-} \in G_{n_{m}}^{-}:=\overline{\operatorname{sp}\left\{e_{n}^{-}: n \geq n_{m}\right\}} \subset L_{w}^{p,-}(\mathbb{R})$ we remark that such a $f^{-}$has a representation

$$
f^{-}=\sum_{n=n_{m}}^{\infty} f_{n}^{-} e_{n}^{-} .
$$

Since this series converges in $L_{w}^{p}(\mathbb{R})$ and every $J^{k}$ is a continuous mapping, (4.13) implies $J^{k} g=0$ for all $k \leq m$. Hence, (4.4) follows from Proposition 4.3.

To complete the proof we remark that the space $L_{w}^{p}(\mathbb{R})$ equals in a natural way the direct sum $L_{w}^{p,-}(\mathbb{R}) \oplus L_{w}^{p,+}(\mathbb{R})$, where the second component is defined as the closed subspace of $L_{w}^{p}(\mathbb{R})$ consisting of functions with supports in $\mathbb{R}^{+}$. The functions

$$
e_{n}^{+}:=e_{n}^{-} \circ \psi \quad, \quad \text { where } \psi(x):=-x \forall x \in \mathbb{R}
$$

form a Schauder basis of $L_{w}^{p,+}(\mathbb{R})$, which plays the same role as the basis $\left(e_{n}^{-}\right)_{n=1}^{\infty}$ has in $L_{w}^{p,-}(\mathbb{R})$. This follows from the formal commutation relations

$$
\partial_{x}^{2}(f \circ \psi)=\left(\partial_{x}^{2} f\right) \circ \psi, e^{t \partial_{x}^{2}}(f \circ \psi)=\left(e^{t \partial_{x}^{2}} f\right) \circ \psi .
$$

Consequently, the union of the sequences $\left(e_{n}^{-}\right)_{n=1}^{\infty}$ and $\left(e_{n}^{+}\right)_{n=1}^{\infty}$ is the desired Schauder basis.

\section{EXAMPLES}

We present some examples of operators $A$ and semigroups generated by $A$ such that the assumptions of Theorems 2.1, 3.1 or 4.1 hold. In general it seems that there is some amount of research to be done in this direction: many more important classes of operators should satisfy our conditions for the higher derivatives of the semigroup kernel, although optimal results in this respect seem not yet to be available in the literature.

$1^{\circ}$. By the classical explicit Gaussian semigroup formula, both Theorem 2.1 and 3.1 can be applied with $M=\infty$ to the case of the Laplacian $A=\Delta$ on $\mathbb{R}^{N}$. 
$2^{\circ}$. The negative bi-Laplacian

$$
A=-\Delta^{2}=-\left(\sum_{j=1}^{N} \partial_{x_{j}}^{2}\right)\left(\sum_{j=1}^{N} \partial_{x_{j}}^{2}\right)
$$

satisfies (3.1) with $M=\infty$. The next argument using the Fourier transform is folklore for specialists in parabolic equations, but since we do not know a reference, we expose a proof for the convenience of the reader. First, the Fourier transform turns the equation (1.1) with $A=-\Delta^{2}$ into

$$
\partial_{t} \widehat{u}(k, t)=-k^{2} k^{2} \widehat{u}(k, t),
$$

hence, solving this elementary ordinary differential equation (where $k$ is just a parameter), taking into account the initial condition (1.2) and applying the inverse Fourier transform shows that the solution of the Cauchy problem (1.1)-(1.2) for the negative bi-Laplacian is given by

$$
\frac{1}{(2 \pi)^{N}} \int_{\mathbb{R}^{N}} \int_{\mathbb{R}^{N}} e^{i k(x-y)} e^{-k^{2} k^{2} t} d k f(y) d y .
$$

Thus, the integral kernel $\widetilde{K}$ of Theorem 3.1 reads as

$$
\widetilde{K}(x, t)=\frac{1}{(2 \pi)^{N}} \int_{\mathbb{R}^{N}} e^{i x k} e^{-k^{2} k^{2} t} d k
$$

We have for all multi-indices $\alpha$

$$
\begin{aligned}
D_{x}^{\alpha} \widetilde{K}(x, t) & =\frac{1}{(2 \pi)^{N}} \int_{\mathbb{R}^{N}}(i k)^{\alpha}\left(\prod_{j=1}^{N} e^{i x_{j} k_{j}} e^{-k_{j}^{2} k_{j}^{2} t} \prod_{\ell \neq j}^{N} e^{-k_{j}^{2} k_{\ell}^{2} t}\right) d k \\
& =: \int_{\mathbb{R}^{N}} k^{\alpha} R(x, k, t) d k .
\end{aligned}
$$

Let us choose an index $j=1, \ldots, N$ and for a moment fix the variables $k_{\ell}$ with $\ell \neq j$ as well as the variables $x$ and $t$. Then, looking at the expression (5.2) one observes that for all $j$ the function

$$
k_{j} \mapsto k^{\alpha} R(x, k, t)
$$

has a unique analytic extension to $\mathbb{C} \ni k_{j}$. In (5.2), the integration with respect to the variable $k_{j}$ can be considered as a path integral along the real axis, and due to the just mentioned analyticity and properties of complex path integrals, the integration contour can be changed into $\gamma_{x, t, j}$, where

$$
\gamma_{x, t, j}\left(k_{j}\right)=k_{j}+i\left|x_{j}\right|^{1 / 3} \operatorname{sgn}\left(x_{j}\right)(L t)^{-1 / 3} \quad \text { for } k_{j} \in \mathbb{R}
$$

$\operatorname{sgn}(a)$ is the sign of the real number $a$ and $L>0$ is a large enough number to be specified soon. For all $k \in \mathbb{R}^{N}$, let

$$
\gamma_{x, t}(k):=\left(\gamma_{x, t, 1}\left(k_{1}\right), \ldots, \gamma_{x, t, N}\left(k_{N}\right)\right) \in \mathbb{C}^{N}, \quad S(x, k, t):=R\left(x, \gamma_{x, t}(k), t\right),
$$

hence,

$$
D_{x}^{\alpha} \widetilde{K}(x, t)=\int_{\mathbb{R}^{N}} \gamma_{x, t}(k)^{\alpha} S(x, k, t) d k
$$


Changing $k_{j}$ into (5.3) for all $j$ causes the following changes in the integrand of (5.2):

$$
\begin{aligned}
e^{i x_{j} k_{j}} \rightarrow & \exp \left(i x_{j} k_{j}-2^{-1}\left|x_{j}\right|^{\frac{4}{3}} L^{-\frac{2}{3}} t^{-\frac{1}{3}}-2^{-1}\left|x_{j}\right|^{\frac{4}{3}} L^{-\frac{2}{3}} t^{-\frac{1}{3}}\right) \\
e^{-k_{j}^{2} k_{j}^{2} t} \rightarrow & \exp \left(-k_{j}^{4} t+4 k_{j}^{2}\left|x_{j}\right|^{\frac{2}{3}} L^{-\frac{2}{3}} t^{\frac{1}{3}}-\left|x_{j}\right|^{\frac{4}{3}} L^{-\frac{4}{3}} t^{-\frac{1}{3}}-i r_{j}(x, k, t)\right) \\
= & \exp \left(-2^{-1} k_{j}^{4} t-2^{-1} k_{j}^{4} t+4 k_{j}^{2}\left|x_{j}\right|^{\frac{2}{3}} L^{-\frac{2}{3}} t^{\frac{1}{3}}-8\left|x_{j}\right|^{\frac{4}{3}} L^{-\frac{4}{3}} t^{-\frac{1}{3}}\right. \\
& \left.+7 x_{j}^{\frac{4}{3}} L^{-\frac{4}{3}} t^{-\frac{1}{3}}-i r_{j}(x, k, t)\right) \\
= & \exp \left(-2^{-1} k_{j}^{4} t-\left(2^{-\frac{1}{2}} k_{j}^{2} t^{\frac{1}{2}}-2^{\frac{3}{2}}\left|x_{j}\right|^{\frac{2}{3}} L^{-\frac{2}{3}} t^{-\frac{1}{6}}\right)^{2}\right. \\
& \left.+7\left|x_{j}\right|^{\frac{4}{3}} L^{-\frac{4}{3}} t^{-\frac{1}{3}}-i r_{j}(x, k, t)\right) \\
e^{-k_{j}^{2} k_{\ell}^{2} t} \rightarrow & \exp \left(-k_{j}^{2} k_{\ell}^{2} t+4 k_{j} k_{\ell}\left|x_{j}\right|^{\frac{1}{3}}\left|x_{\ell}\right|^{\frac{1}{3}} L^{-\frac{2}{3}} t^{\frac{1}{3}}\right. \\
& \left.-\left|x_{j}\right|^{\frac{2}{3}}\left|x_{\ell}\right|^{\frac{2}{3}} L^{-\frac{4}{3}} t^{-\frac{1}{3}}-i r_{j, \ell}(x, k, t)\right) \\
= & \exp \left(-\left(k_{j} k_{\ell} t^{\frac{1}{2}}-2\left|x_{j}\right|^{\frac{1}{3}}\left|x_{\ell}\right|^{\frac{1}{3}} L^{-\frac{2}{3}} t^{-\frac{1}{6}}\right)^{2}\right. \\
& \left.+3\left|x_{j}\right|^{\frac{2}{3}}\left|x_{\ell}\right|^{\frac{2}{3}} L^{-\frac{4}{3}} t^{-\frac{1}{3}}-i r_{j, \ell}(x, k, t)\right)
\end{aligned}
$$

where all $r_{j}$ and $r_{k, \ell}$ are some real valued functions which we do not need to specify. We need to evaluate $|S(x, k, t)|$; our aim is to prove the upper bound

$$
|S(x, k, t)| \leq e^{-b|k|^{4} t-b\left(|x|^{4} t^{-1}\right)^{1 / 3}}
$$

for it, where $b>0$ is some constant. To this end we can omit all imaginary terms of the exponent of $S(x, k, t)$. Moreover, choosing $L>1$ large enough so that $L^{-2 / 3}>>L^{-4 / 3}$, half of the negative last term $-2^{-1}\left|x_{j}\right|^{\frac{4}{3}} L^{-\frac{2}{3}} t^{-\frac{1}{3}}$ of $(5.5)$ cancels out the positive term $7\left|x_{j}\right|^{\frac{4}{3}} L^{-\frac{4}{3}} t^{-\frac{1}{3}}$ in (5.6). In the same way, using

$$
\sum_{j=1}^{N}\left|x_{j}\right|^{2} \geq \frac{1}{2 N^{2}} \sum_{j=1}^{N} \sum_{\ell=1}^{N}\left|x_{j} x_{\ell}\right|
$$

and possibly enlarging $L$, the positive term in (5.7) can still be cancelled out by the last term of (5.5). The estimate (5.8) follows from the remaining negative terms $-2^{-1}\left|x_{j}\right|^{\frac{4}{3}} L^{-\frac{2}{3}} t^{-\frac{1}{3}}$ in $(5.5)$ and $-2^{-1} k_{j}^{4} t$ in (5.6).

Using (5.4), (5.8) we estimate for all $t \geq 1$ and all $\alpha \in \mathbb{N}_{0}^{N}$

$$
\begin{aligned}
\left|D_{x}^{\alpha} \widetilde{K}(x, t)\right|= & \frac{1}{(2 \pi)^{N}}\left|\int_{\mathbb{R}^{N}} \gamma_{x, t}(k)^{\alpha} S(x, k, t) d k\right| \\
\leq & C_{L} \int_{\mathbb{R}^{N}}\left(|k|^{|\alpha|}+\left(|x| t^{-1}\right)^{\frac{|\alpha|}{3}}\right) e^{-b|k|^{4} t-b\left(|x|^{4} t^{-1}\right)^{1 / 3}} d k \\
\leq & C_{L} e^{-b\left(|x|^{4} t^{-1}\right)^{1 / 3}} \int_{\mathbb{R}^{N}}|k|^{|\alpha|} e^{-b|k|^{4} t} d k \\
& +C_{L}\left(|x| t^{-1}\right)^{\frac{|\alpha|}{3}} e^{-b\left(|x|^{4} t^{-1}\right)^{1 / 3}} \int_{\mathbb{R}^{N}} e^{-b|k|^{4} t} d k
\end{aligned}
$$




$$
\begin{aligned}
\leq & C_{L}^{\prime} e^{-b\left(|x|^{4} t^{-1}\right)^{1 / 3}} t^{-\frac{1}{4}|\alpha|} \int_{\mathbb{R}^{N}}\left(t^{\frac{1}{4}}|k|\right)^{|\alpha|} e^{-b|k|^{4} t} d k \\
& +C_{L}^{\prime} t^{-\frac{3|\alpha|}{4}}\left(|x| t^{-1 / 4}\right)^{\frac{|\alpha|}{3}} e^{-b\left(|x|^{4} t^{-1}\right)^{1 / 3}} \\
\leq & C_{L}^{\prime \prime} t^{-\frac{1}{4}|\alpha|} e^{-b^{\prime}\left(|x|^{4} t^{-1}\right)^{1 / 3}}
\end{aligned}
$$

for some constant $0<b^{\prime}<b$. The $L^{2}$-norm of this function has the bound $C t^{-\frac{|\alpha|}{4}+\frac{N}{4}}$ for $t \geq 1$, which is of the form (3.1).

$3^{\circ}$. Other examples: Theorem 4.1 applies for example in much more complicated cases like the linearized Cahn-Hilliard equation, see the semigroup estimates in Th.2.1 of [3], where the kernel satisfies the estimate (4.1) with $M=3, a=1 / 3$, $b=2 / 3$.

\section{REFERENCES}

[1] J. Bonet, W. Lusky, J. Taskinen, Schauder bases and the decay rate of the heat equation. J. Evolution Eq. 19 (2019), 717-728.

[2] D. Gilbarg, N. Trudinger, Elliptic partial differential equations of second order, SpringerVerlag, Berlin-Heidelberg, 2001.

[3] P. Howard, Linear stability for transition front solutions in multidimensional Cahn-Hilliard systems. J.Dyn. Diff. Equat. 29 (2017), 895-955.

[4] O. Ladyszenskaya, The boundary value problems in mathematical physics, Springer-Verlag, New York-Berlin-Heidelberg-Tokyo, 1985

[5] J. Lindenstrauss, L. Tzafriri, Classical Banach spaces I, Springer-Verlag, Berlin, 1977.

[6] J. Nečas, Direct methods in the theory of elliptic equations. Springer Monographs in Mathematics. Springer, Heidelberg, 2012.

[7] A. Pazy, Semigroups of linear operators and applications to partial differential equations, Springer-Verlag, New York, 1983.

[8] W. Rudin, Functional Analysis, 2nd edition, McGraw-Hill Science/Engineering/Math, New York, 1991.

[9] P. Wojtaszyck, Banach spaces for analysts. Cambridge Studies in Advanced Mathematics vol. 25, Cambridge University Press, Cambridge, 1991.

\section{Authors' addresses:}

José Bonet: Instituto Universitario de Matemática Pura y Aplicada IUMPA, Universitat Politècnica de València, E-46071 Valencia, Spain email: jbonet@mat.upv.es

Wolfgang Lusky: Institut für Mathematik, Universität Paderborn, D-33098 Paderborn, Germany.

email: lusky@uni-paderborn.de

Jari Taskinen: Department of Mathematics and Statistics, P.O. Box 68, University of Helsinki, 00014 Helsinki, Finland.

email: jari.taskinen@helsinki.fi 